\title{
Cross-cultural adaptation and psychometric properties of the MMSE and MoCA questionnaires in Tanzanian Swahili for a traumatic brain injury population
}

Joao Ricardo Nickenig Vissoci ${ }^{1,2,3^{*}}$ (D, Leonardo Pestillo de Oliveira ${ }^{4}$, Temitope Gafaar ${ }^{5}$, Michael M. Haglund ${ }^{1,3}$, Mark Mvungi ${ }^{6}$, Blandina Theophil Mmbaga ${ }^{6,7}$ and Catherine A. Staton ${ }^{1,2,3}$

\begin{abstract}
Background: Traumatic Brain Injury (TBI) is the most common cause of injury-related death and disability globally, and a common sequelae is cognitive impairment. Addressing post-TBI cognitive deficits is crucial because they affect rehabilitation outcomes, but doing this requires valid and reliable cognitive assessment measures. However, no such instrument has been validated in Tanzania's TBI population. Mini-Mental State Examination (MMSE) and Montreal Cognitive Assessment (MoCA) are two commonly used instruments to measure cognitive impairment, and there have been a few studies reporting their use in post-TBI cognitive assessment. Our aim was to report the psychometric properties of the Swahili version of both scales amongst the TBI population in Tanzania.

Methods: A cross-cultural adaptation committee participated in the translation and content validation process for both questionnaires. Our patient sample consisted of 192 adults with TBI who were admitted to Kilimanjaro Christian Medical Center (KCMC) in Tanzania. Confirmatory factor analysis, reliability and external validity were evaluated.

Results: MoCA showed adequate factor loadings (values $>0.50$ for all items except items $7 \& 10$ ) and adequate reliability (values $>0.70$ ). Factor loadings for most of the MMSE items were below 0.5 and internal consistency was medium (<0.7). Polychoric correlation between MMSE and MoCA was strong, positive and statistically significant $(r=0$. $68, p=0.001)$; correlation with the cognitive subscale of FIM indicated moderately positive relationships - MMSE $(r=0$. $35, p=0.001)$ and MoCA $(r=0.43, p=0.001)$.

Conclusions: With the exception of the language and memory items, MoCA is a valid and reliable instrument for cognitive impairment screening in Tanzania's adult TBI population. On the other hand, MMSE does not appear to be an appropriate tool in this patient group, but its positive correlations with MoCA and CFIM indicate similar theoretical concepts. Both instruments require further validation studies to prove their predictive ability for screening cognitive impairment before they are considered suitable for clinical use.
\end{abstract}

Keywords: Tanzania, Traumatic brain injury, Cognitive impairment, MoCA, MMSE, Validity, Reliability

\footnotetext{
* Correspondence: jnv4@duke.edu

'Duke Global Health Institute, Duke University, Durham, NC, USA

2Duke Emergency Medicine, Duke University School of Medicine, Durham,

NC 27710, USA

Full list of author information is available at the end of the article
}

(c) The Author(s). 2019 Open Access This article is distributed under the terms of the Creative Commons Attribution 4.0 International License (http://creativecommons.org/licenses/by/4.0/), which permits unrestricted use, distribution, and reproduction in any medium, provided you give appropriate credit to the original author(s) and the source, provide a link to the Creative Commons license, and indicate if changes were made. The Creative Commons Public Domain Dedication waiver (http://creativecommons.org/publicdomain/zero/1.0/) applies to the data made available in this article, unless otherwise stated. 


\section{Introduction}

Of all injuries, traumatic brain injury (TBI) is one of the most common causes of death and disability globally, and is expected to surpass many diseases as a major cause of death and disability by 2020 [1]. An estimated 10 million people are affected annually [2,3] with over 57 million people worldwide hospitalized with one or more TBI [4]. Its sequelae include: changes in cognition, short term memory loss, attention deficit, mood disturbances, and personality changes - including impulsivity and irritability [5-16]. In particular, cognitive impairment due to TBI is a substantial source of morbidity for affected individuals, their family members, and communities at-large [17]. Deficits in attention, memory, and executive functioning are the most common neurocognitive consequences of TBI at all levels of severity $[5,18]$. Especially problematic are impairments to relatively basic cognitive functions, such as attention and memory because these may cause or worsen additional deficits in executive function, communication, and other more complex processes [19]. Addressing post-TBI cognitive deficits is crucial as cognitive impairment is an important factor that affects rehabilitation outcomes [20, 21]. Hence, thorough neuropsychiatric assessment, including cognitive assessment, is essential to guiding rehabilitation efforts and appropriate medication regimens [22].

Two widely used tools for assessing cognitive function are the Mini-Mental State Examination (MMSE) and the Montreal Cognitive Assessment (MoCA). MMSE was originally developed in the United States in 1975 for dementia screening [23]. The scale assesses cognitive capacities with respect to orientation, registration, attention, recall, and language, as well as the ability to follow verbal and written commands [23]. It is probably the most popular measure to screen for cognitive impairment and has been culturally validated in many countries. While the scale is helpful in approximating gross cognitive ability, there are concerns that it does not take into account mental elasticity and working memory, [24] and that it has ceiling effects when administered to individuals with high educational circumstances [25]. The MoCA, developed in 2005, is another widely used measure of cognitive function [26]. It comprises 10 items, with varying degrees of difficulty, which assess 6 cognitive domains: executive functioning; visuospatial abilities; short-term memory; language; attention, concentration, and working memory; and temporal and spatial orientation [26]. Values for the for MMSE and MoCA are similar - 0 to 30 range, with higher scores indicating better cognitive functioning. Similar to MMSE, MoCA is also used for evaluating cognitive impairment in early dementia, but it has been shown to have greater sensitivity than MMSE when screening for mild cognitive impairment
(MCI), mild dementia, and cognitive impairment resulting from stroke [27].

Specific to TBI, both MoCA and MMSE have been used to measure patient-reported post-TBI cognitive outcomes [20, 28, 29]. There have been a few studies that have evaluated the use of MoCA in these patients; Wong et al. validated the scale in TBI patients with intracranial hemorrhage in Hong Kong [29] and Kumar et al. tested the reliability of MoCA in screening for mild TBI. Zhang et al. reported on the sensitivity of MMSE in screening for post-TBI cognitive impairment. However, to our knowledge, there have been no studies reporting the construct validity and reliability of MMSE in TBI patients.

At $\mathrm{KCMC}$, a regional referral hospital in northern Tanzania, TBI is the leading cause of injury-related death and disability and it represents $\sim 6 \%$ of all emergency department visits [30]. Post-TBI cognitive impairment is often assessed using MMSE and MoCA since both scales are short, easy to administer, and feasible to implement as cognitive screeners. However, to date, neither tool has been psychometrically tested in Tanzania - or in its TBI population. The importance of doing this cannot be emphasized enough. For instance, due to the fact that both tools were developed in English, a recurring issue when translating these scales is that the prompts involving language abilities have had to be adapted to reflect culturally appropriate constructs. For example, the MMSE construct, "No ifs, ands, or buts" has often been replaced as no direct translation for this phrase exists in many languages [31, 32]. The phrase "Close your eyes" which is one of the command prompts in MMSE, means death in Chinese in certain contexts; so, some researchers have changed the prompt to "Raise your hands" [31, 32].

Despite the current use of MMSE and MoCA at KCMC, the Swahili versions of these scales - or any other scale - have not been validated to screen for post-TBI cognitive impairment in Tanzania, where Swahili is the primary language. This represents a deficit in the capacity to appropriately diagnose and treat post-TBI cognitive morbidity. Therefore, the aims of this study were to (a) develop the first systematic translation and adaptation of MMSE and MoCA in Tanzanian Swahili and (b) to analyse the psychometric properties of the scales in Tanzanian TBI patients, including evidence of reliability, construct validity, and external validity.

\section{Methods}

\section{Study design and setting}

Moshi, a municipality in the Kilimanjaro region of Northern Tanzania with a population of 184,292 , is home to Kilimanjaro Christian Medical Centre (KCMC), the third largest hospital in the country and the referral hospital for northwestern Tanzania [33]. 


\section{Participants}

Patients who met the inclusion criteria - being older than 18 years of age, seeking acute care for a TBI of any severity, being admitted for continued care, being able to speak and understand Swahili, being able to answer questions adequately and providing consent to participate before discharge - were approached for enrollment into this study. After going through the informed consent process, participants were enrolled in the KCMC TBI registry prior to hospital discharge. Patients responded to the Tanzanian versions of the MMSE and the MoCA at the bedside along with a longer set of mental health and functioning interview questions. Quality control for the data collected and entered was performed by the principal investigator. Questionnaire responses were gathered and stored using REDCap [34]. Further details about the KCMC TBI registry methodology can be found elsewhere [30].

\section{Translation and adaptations}

The translation and cross-cultural adaptation committee was constituted of five bilingual researchers or research nurses who voluntarily participated in the translation, adaptation and content validation of both instruments. With the adapted translated version, we piloted the instrument with a convenience sample of 20 adult paticipants from Tanzania. The pilot test allowed us to verify the quality and the questions, and the content and language coherence of the instrument [35].

As suggested by the WHO for health outcomes translation [36], both instruments underwent our independent back translation protocol: i) native bilingual Swahili translators were hired to independently translate MMSE and MoCA into Swahili; ii) then bilingual language translators were hired to back-translate the Swahili versions to English; iii) the English back-translations were then compared with the original versions of the instruments and inconsistencies were checked by two independent bilingual researchers; and iv) the last step was to evaluate for issues with semantics and make adjustments as deemed appropriate by the researchers. Swahili and English versions of both scales are included as additional files with the current publication (Additional files 1, 2, 3 and 4).

To analyze the theoretical and content evaluation of the translated instruments, a set of focus group discussions were conducted with expert judges. This served to verify: (a) the practical relevance and (b) language clarity of the translated instruments, and (c) the theoretical coherence of the items. The experts' opinions were discussed collectively in the focus groups sessions to address discordance and improve translation quality.

\section{Data analysis}

Patient characteristics were reported using descriptive statistics (mean, standard deviations, medians, interquartile range and frequency distributions). All analyses were performed using the $\mathrm{R}$ Language for Statistical Computing software [37].

\section{Internal validity}

To verify the construct validity of MMSE and MoCA, Confirmatory Factor Analysis (CFA) was employed, based on the prior literature looking at the factor structure in different populations. CFA is a statistical technique that is used to evaluate the appropriateness of a measurement model that is derived from prior empirical research and/or theory [38]. For both of these scales, previous studies that have analyzed the construct validities have differed in the reported factor structures. For MMSE, some studies have reported two factors [39-41]; or five factors [42]. Some studies have reported MoCA with 7 factors [43]; 6 factors [26, 44]; or 2 factors [45]. Since the purpose of this study is to evaluate MMSE and MoCA as cognitive screening tools, the scales were analyzed unidimensionally as has been done in some prior studies - Jones \& Gallo et al. for MMSE [46]; Freitas et al. and Janelidze et al. for MoCA [44, 47].

Confirmatory Factor Analysis model adequacy was tested using commonly accepted indices intended to evaluate model fit [38, 48]. Weighted Least Square Means and Variance Adjusted (WLSMV). The following fit indices were used to test model adjustment: Chi-square ( $\mathrm{X}^{2}$ and $p$-value), Root Mean Square Error of Approximation (RMSEA <0.08, CI 95\%), Tucker-Lewis index (TLI $>0.95)$, and Comparative Fit Index (CFI > 0.95). We also calculated the Average Variance Extracted (AVE) with values greater than 0.50 were considered satisfactory indicators of construct validity [49].

\section{Reliability}

In order to test the internal consistency of the items, the Cronbach's alpha, Omega and Composite Reliability analysis were performed for both instruments, considering that coefficients above 0.7 are acceptable [50].

\section{External validity}

We tested external validity in two ways.

i. By correlating MMSE and MoCA with each other as was done in previous studies, which showed a high level of correlation between both scales [43, 51-55].

ii. By correlating MMSE and MoCA with the cognitive subscale of Functional Independence Measure (cFIM). FIM is a widely-used scale designed to determine an individual's level of disability, as reflected by the need for assistance [56]. Higher FIM scores indicate higher levels of independence, and the scale can be divided into the motor subscale and the cognitive subscale. Prior studies have shown positive correlations (moderate 
to strong) between MMSE, MoCA and cFIM $[21,55,57]$; so we also administered FIM to our study sample.

Our hypothesis was that the Swahili, adapted versions of MMSE and MoCA questionnaires would correlate positively with each other and with the cognitive subscale of FIM, confirming the instruments' ability to behave as expected in relation to their theoretical concepts.

\section{Results}

Study participants included 192 adults, all of whom were part of KCMC's TBI patient registry and posthospitalization cohort study. Most of the participants were male $(82.8 \%)$ and overall had an average age of 33.87 ( $\mathrm{SD}=13.32$ ) years old; this is consistent with previously reported demographic information on TBI patients at KCMC [30]. In relation to injury, most patients (91\%) had a mild TBI severity, with a Glasgow Coma Score of 13-15. Full demographic and health status characteristics of the study participants are presented in Table 1. Descriptive analysis showed that participants endorsed to all the response possibilities for each of the 11 items of MMSE and the 10 items of MoCA.

\section{Internal validity \& reliability}

Using CFA, both scales were analyzed uni-dimensionally, and model adequacy was tested. The fit indices indicated that a unidimensional model was adequate for MoCA but less so for MMSE (Table 2). Factor loadings were below 0.5 for 7 of the 11 items in MMSE (items 3, 5, 6, 7, 9, 10, \& 11), while the rest were adequate (Fig. 1); AVE for the scale was 0.16 (Table 2). Regarding MMSE's internal consistency, medium scores were found (below 0.7) for all the tested reliability measures (Table 2). The MoCA questionnaire showed adequate factor loadings (above 0.50) for all items, except for items 7 \& 10 (Fig. 1); AVE was 0.40 (Table 2). MoCA's internal consistency was adequate with scores above 0.70 for all reliability measures tested (Table 2).

Invariance analysis was performed for MoCA - the subjects were divided into 2 groups based on education level ('some primary education' and 'more than primary education'). Overall, MoCA showed evidence of configural effects and intercept invariance in relation to education level. However, when the analysis was performed using the factor means model, the scale showed variance with respect to education. Specifically, for the items m7 (language) and m10 (memory), participants with some primary education performed worse than participants who had more than primary-level education. This variance was not observed when the configural model analysis was performed. Invariance analysis was not performed for the MMSE because the values of fit and its factor loadings indicated model inadequacy (Table 2).

\section{External validity}

MMSE showed a strong positive polychoric correlation $(>0.5)$ with MoCA $(r=0.68, p=0.001)$ (Table 3, Fig. 2). When correlated with cFIM, both scales demonstrated moderately positive relationships - MMSE $(r=0.35$

Table 1 Baseline characteristics of the validation sample

\begin{tabular}{lc}
\hline VARIABLES & 33.87 (13.32) \\
\hline Age (years), Mean (SD) & $4.43(2.48)$ \\
Household size, Mean (SD) & $\$ 67.11(26.8 ; 134.2)$ \\
Monthly personal income, USD, Median (IQR) & $\$ 98.4(44.7 ; 156.6)$ \\
Monthly family income, USD, Median (IQR) & $159(82.8)$ \\
Male, N (\%) & $104(54.7)$ \\
Married, N (\%) & $44(21.7)$ \\
Occupation, N (\%) & $41(22.3)$ \\
Business & $23(12.5)$ \\
Farming & $67(36.4)$ \\
Skilled worker & $13(7.1)$ \\
Salaried worker & $112(59.3)$ \\
Other & $44(23.3)$ \\
Education, N (\%) & $33(17.5)$ \\
$\quad$ Some primary education & \\
Some secondary education & $91 \%$ \\
Some university education & \\
Severity of Injury & \\
\hline
\end{tabular}


Table 2 Psychometric properties for content and construct validity

\begin{tabular}{lll}
\hline & MMSE & MoCA \\
\hline Reliability & & \\
Cronbach's Alpha & $0.63(0.60 ; 0.66)$ & $0.78(0.73 ; 0.80)$ \\
KMO & 0.65 & 0.80 \\
Composite Reliability & 0.64 & 0.86 \\
CFA & & \\
$X^{2}$ (Df) / p-value & $66.27(33) / 0.001$ & $46.80(45) / 0.08$ \\
RMSEA (Cl 95\%) & $0.06(0.04 ; 0.08)$ & $0.04(0.00 ; 0.07)$ \\
TLI & 0.81 & 0.98 \\
CFI & 0.85 & 0.98 \\
Factor loadings range & $0.00-0.60$ & $0.30-0.81$ \\
Average extracted variance & 0.16 & 0.4
\end{tabular}

KMO Kaiser-Meyer-Olkin coefficient, CFA Confirmatory Factor Analysis, $X^{2}$ ChiSquare, Df Degree of Freedom, RMSEA Root Mean Square Error of

Approximation, TLI Tucker-Lewis Index, CFI Comparative Fit Index

$p=0.001)$ and MoCA $(r=0.43, p=0.001)$. Table 3 contains the correlation coefficients between MMSE, MoCA and cFIM - the subscale aggregate and its individual items. With the exception of two items comprehension and memory - which were more positively correlated with MMSE, the items in cFIM had higher positive coefficients when correlated with MoCA than when correlated with MMSE. This confirms the instruments' ability to behave as expected in relation to the theoretical concept.

\section{Discussion}

The purpose of this study was to evaluate the psychometric properties of the Tanzanian Swahili versions of MMSE and MoCA as screening tools for post-TBI cognitive impairment in the country. This is the first study to report on the scales' properties in Tanzania, and on the internal consistency and construct validity of MMSE in TBI patients. The Tanzanian version of MoCA showed satisfactory psychometric properties, with adequate reliability and content and construct validity (except for two items). MMSE, on the other hand, performed poorly; although its content validity was adequate, its reliability scores were medium, and factor loadings for most of its items were unsatisfactory. Pertaining to external validity, the significantly high level of positive correlation between MMSE and MoCA is consistent with previous literature $[43,51-54]$ and indicates that on a gross level, both of these instruments measure similar theoretical concepts with regards to cognitive deficits. In addition, our study found a positive correlation between MMSE, MoCA and cognitive subscale of FIM - similar to what has been reported in the literature $[21,55,57]$ - providing further evidence of the external validity of both scales.
Regarding factor structure, MoCA was originally designed to have 6 dimensions: executive functioning; visuospatial abilities; short-term memory; language; attention, concentration, and working memory; and temporal and spatial orientation [26]. Exploratory analysis of MMSE demonstrated 5 robust dimensions: concentration; language/praxis; orientation; attention; and memory [42]. Functionally, however, both the MMSE and the MOCA are usually scored as a unidimensional construct, with single total score providing a global assessment of cognition [58]. Similar to prior studies that have found the MoCA to be a good MCI screening tool $[44,47]$, our internal structure analysis of the Swahili version of MoCA found the unidimensional model to demonstrate excellent fit. On the other hand, fitness indices indicated inadequacy in utilizing the Swahili MMSE in a unidimensional fashion.

\section{MoCA and TBI}

Prior to this study, MoCA was validated in TBI patients with intracranial hemorrhage in Hong Kong [29]. Kumar et al. tested its reliability in screening for mild TBI and found an $87.9 \%$ sensitivity and a $66.7 \%$ specificity for detecting cognitive impairment. Therefore, prior literature recommend that the MoCA could be an effective tool to guide rehabilitation and treatment efforts [59]. Our results support these previous results; we found that the Tanzanian MoCA demonstrates adequate psychometrics in TBI patients.

With the exception of two items, the Tanzanian MoCA showed good content and construct validity. This was consistent with prior MoCA validation studies in Egypt, China, Chile, Japan, Colombia, Sri Lanka, Korea, and Brazil [43, 51-54, 60-62]. Regarding the two items (language and memory) that had unsatisfactory factor loadings, invariance analysis indicates that the education level of subjects must be taken into account and highlights the importance of analysing the scale results at an aggregate level and, also, at an individual item level. The reliability scores for each factor met internal consistency criteria - equal to or higher than $0.70[49,63]$ - and were also consistent with the existing literature $[43,51-54,60-62]$. However, given that the Cronbach's alpha has been criticized in the psychometrics literature, we also calculated alternative reliability scores (Composite Reliability and Omega coefficients). Both scores have shown to be consistent with reduced bias [64]. In our resulrs, all reliability coefficients (Alpha, Composite Reliability, and Omega) confirmed satisfactory reliability of the Swahili version of MoCA [64].

There have been a few studies that have validated post-TBI cognitive assessment tools - these include 

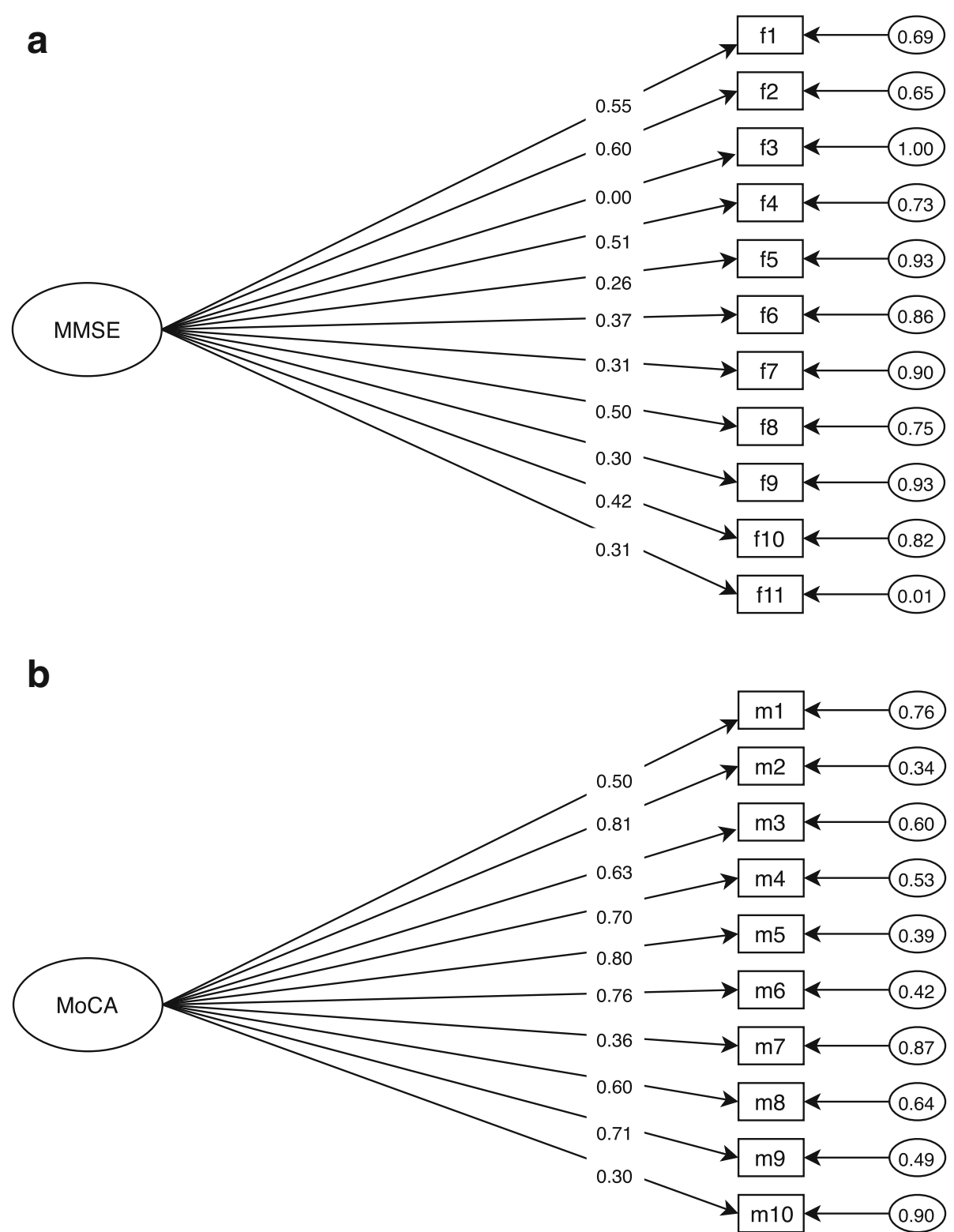

Fig. 1 Confirmatory factor analysis diagrams, factor loadings for MMSE and MoCA. Numbers on the left of the items indicate the factor loadings while numbers on the right indicate error terms

Brain Injury Alert screening tool (BI Alert) [65] and Immediate Post Concussion Assessment and Cognitive Testing (ImPACT). However, unlike MoCA, which is widely used and has been translated and validated in many languages, these tools are not as widely available and limited evidence exists on their generalizability to other cultures or population subsets. External validity was assessed by comparing the performance of MoCA to MMSE and cFIM, but there is no established gold standard tool for measuring cognitive impairment in Tanzania. So, even though our results preliminarily verify the cross-cultural adaptation and validation of MoCA amongst Tanzania's TBI patient population, more research is needed to establish its performance when compared with other measures of post-TBI cognitive impairment.

\section{MMSE and TBI}

Despite the lack of prior validation studies of MMSE in Tanzania, it is often used to screen for cognitive deficits in health institutions. This is the first study to report on the psychometric properties of the Tanzanian Swahili version of MMSE, as well as the first study to report on its internal consistency and construct validity in 
Table 3 Correlation between MMSE, MoCA and CFIM

\begin{tabular}{lll}
\hline & MMSE (R) & MOCA (R) \\
\hline MMSE & 1 & 0.68 \\
MOCA & 0.68 & 1 \\
CFIM & 0.35 & 0.43 \\
CF17- Expression & 0.34 & 0.38 \\
CF18- Comprehension & 0.46 & 0.43 \\
CF19 - Reading & 0.43 & 0.57 \\
cF20 - Writing & 0.41 & 0.49 \\
CF21 - Speech intelligibility & 0.33 & 0.47 \\
CF22 - Social Interaction & 0.31 & 0.42 \\
CF23 - Emotional Status & 0.27 & 0.31 \\
CF24 - Adjustment to limitations & 0.26 & 0.37 \\
CF25 - Use of leisure time & 0.24 & 0.41 \\
cF26 - Problem Solving & 0.25 & 0.35 \\
cF27 - Memory & 0.36 & 0.26 \\
cF28 - Orientation & 0.28 & 0.33 \\
cF29 - Concentration & 0.37 & 0.41 \\
CF30 - Safety awareness & 0.34 & 0.36 \\
\hline
\end{tabular}

post-TBI patients. Our results indicate that, even though the scale has been used globally and in Tanzania to monitor TBI symptoms [28, 66-70], the Swahili version of MMSE is not a good cognitive impairment screening for this population. The scale has also been found to be have less sensitivity in screening for post-TBI cognitive impairment than MoCA [20]. However, as stated earlier, MMSE's strong correlation with MoCA, and its moderate correlation with CFIM, provide evidence that these scales evaluate similar concepts. MMSE's poor psychometric properties indicate scale instability and high measurement error. As such, further adaptation studies and exploratory factor analyses may be appropriate to investigate how the Tanzanian Swahili version of MMSE could be adjusted and improved for post-TBI cognitive evaluation.

\section{Limitations}

Results of this study should be taken in the context of its limitations. One limitation with this study is related to its sample, being that only TBI patients were included; however, this is also a strength of this study. Even though validation studies are usually conducted with more general populations, there is a need to evaluate the psychometric properties of these instruments in high-risk patient samples such as ours - TBI is the leading cause of death and disability due to injury at KCMC. Our results demonstrate that MoCA can provide relevant diagnostic value to help healthcare professionals in comprehending acquired cognitive deficits among TBI patients. Nevertheless, this sample is unlikely to be representative of the entire adult Tanzanian population, so further studies are warranted to evaluate the psychometric properties of the Swahili versions of the tests in the general Tanzanian population. Secondly, TBI disproportionately affects young males, in Tanzania and globally, and this is reflected in our imbalanced sample. Performances on MMSE and MoCA scales are often associated with certain demographic factors, which is the reason validation studies such as ours are typically required. However, the limited variability in our study sample means we were not able to test the influence of gender and age. Thus, applying age- and gender-based comparisons should be considered with caution.

Another factor to be considered is that $\mathrm{m} 10$, the one of the MoCA items that measures memory, had an inadequate factor loading, and memory is one of the most commonly impaired domains in TBI [17]. A possible explanation is the fact that the item was shown to be influenced by the education level of subjects and majority of our TBI patient sample only had "some primary education". Another potential study limitation is the usage of MMSE in this context. While we included the MMSE due to its widespread use in post-TBI cognitive assessments, some prior literature suggests a neuroanatomical discrepancy between regions affected by TBI and those assessed by the MMSE [71]. Ultimately, our data suggest challenges with using the scale for post-TBI cognitive assessment in our setting but we can't predict the cause of those challenges. Finally, we could not include criterion validity estimates in our study, which denotes a potential limitation to the clinical interpretation of our results. We were unable to identify current valid and reliable clinical measures for cognitive functioning in the Tanzanian culture because we were unable to include a neurologist's assessment of our participants cognitive functioning to serve as the gold standard to allow us to calculate sensitivity and specificity and cutoff points of MoCA. Unfortunately, there is a gap in healthcare capacity related to neurology and psychiatry in Tanzania to support adequate cognitive screening, or to train healthcare providers to provide screening. As a step toward increasing capacity to assess for cognitive impairment, the current study aimed to evaluate the translation and adaptation of MMSE and MoCA to Swahili. Given that MoCA has been shown to be psychometrically adequate, we suggest that its criterion validity should be the subject of future work.

\section{Conclusion}

Cognitive impairment is a major cause of TBI-related disabilities and an important factor that affects rehabilitation outcomes. Yet, there have been a limited number of studies that have evaluated the psychometric 


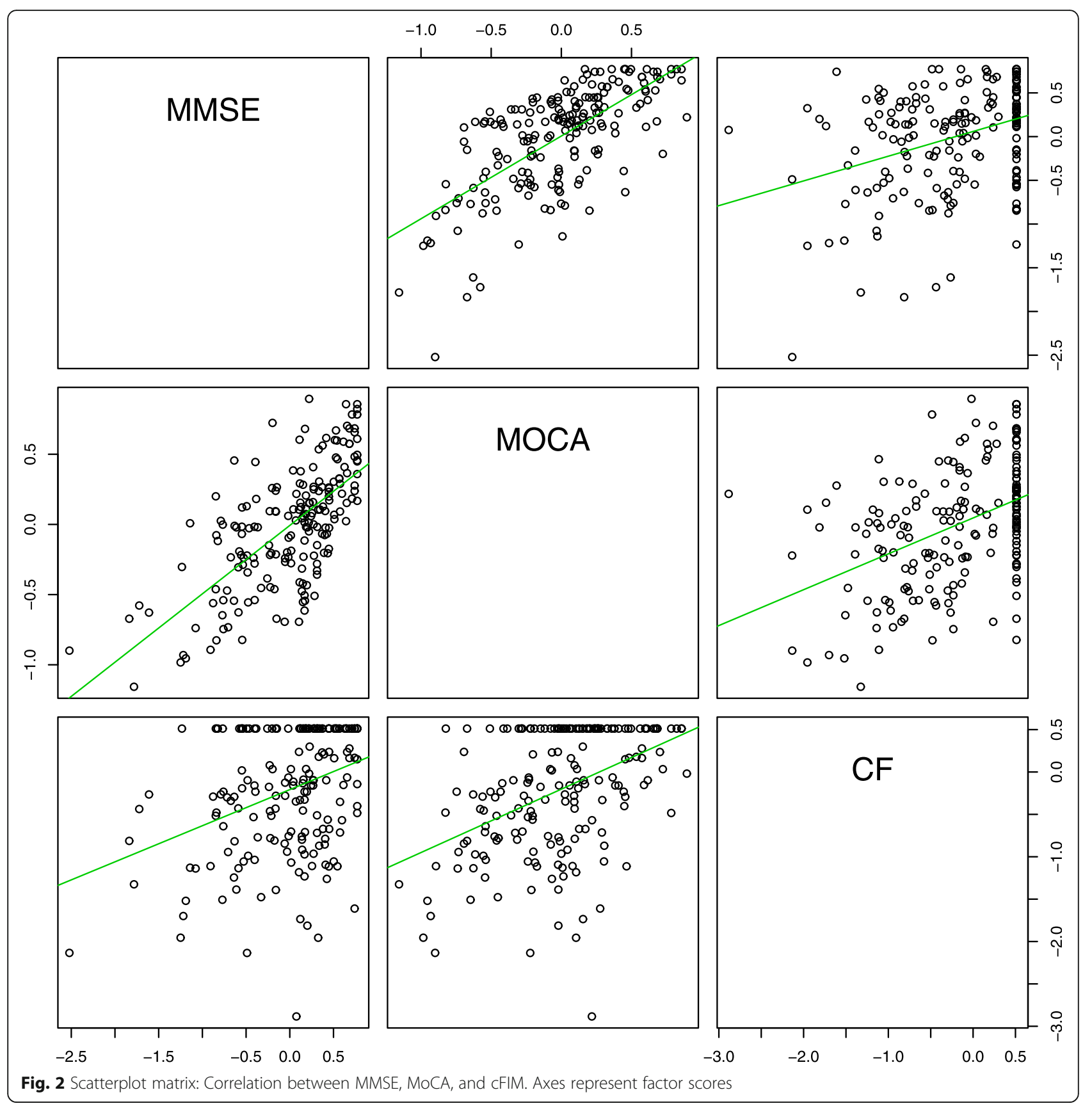

properties of MMSE and MoCA, two widely used cognitive screening tools in TBI patients in sub-Saharan Africa and around the globe. This is an initial report on the reliability and validity of these scales in a Tanzanian TBI patient sample. With the exception of the language and memory items, which are influenced by education level, MoCA is a valid and reliable instrument to screen for cognitive impairment in this population. MMSE does not appear to be a good cognitive screening tool in this patient sample; however, its positive correlation with MoCA and cFIM indicate similar theoretical concepts. This cross-cultural validation of MoCA further develops the capacity to screen for, measure, and treat disorders of cognitive functioning in Tanzania's TBI patients, as well as allowing for more evidence-based practices and advances in research and policy development.

\section{Additional files}

Additional file 1: Tanzanian Swahili version of MMSE. (DOCX $25 \mathrm{~kb}$ ) Additional file 2: Tanzanian Swahili version of MoCA. (DOCX $130 \mathrm{~kb}$ ) Additional file 3: English version of MMSE. (DOCX $21 \mathrm{~kb}$ )

Additional file 4: English version of MoCA. (DOCX $130 \mathrm{~kb}$ ) 


\section{Abbreviations}

CFA: Confirmatory Factor Analysis; FIM/cFIM: Functional Independence Measure/Cognitive subscale of Functional Independence Measure; KCMC: Kilimanjaro Christian Medical Center; LMIC: Low-and Middle-Income Country; MCl: Mild cognitive impairment; MMSE: Mini-Mental State Examination; MoCA: Montreal Cognitive Assessment; TBI: Traumatic brain injury; WHO: World Health Organization

\section{Acknowledgements}

We would like to acknowledge our KCMC/Duke ED Research Team without whom none of our research would be possible.

\section{Funding}

This project was made possible by the Mentored Research Training Program in collaboration with the HRSA-funded KCMC MEPI grant \# T84HA21123-02; U.S. National Institutes of Health and the Duke Division of Emergency Medicine. Dr. Staton would like to acknowledge salary support provided by the Fogarty International Center of the National Institutes of Health under Award Number K01TW010000 (PI, Staton). Funding bodies were not involved in the design of the study, data collection, analysis, interpretation or writing the manuscript.

\section{Availability of data and materials}

The datasets used and/or analyzed during the current study are available from the corresponding author on reasonable request.

\section{Authors' contributions}

JRNV, CAS, BTM conceived of the study. CAS, MM, BTM oversaw translation, adaptation and data collection. LPO, JRNV, TG analyzed the data. MMH, BTM, MM, CAS, JRNV interpreted the data. TG wrote the first draft of the manuscript. All authors edited and approved the final version of the manuscript.

\section{Ethics approval and consent to participate}

The study was approved by the Institutional Review Board of Duke University (IRB \#Pro000061652), the Ethics Committee of the Kilimanjaro Christian Medical Center (821/2018), and the National Institute for Medical Research of Tanzania. Written and verbal consent was obtained from all participants in their native language (Kiswahili). When participants were not capable of signing, written consent was obtained by fingerprints. The ability to provide consent was considered an eligibility criteria. All participants were deemed capable of providing consent by their primary clinician. Participants ability to provide consent was evaluated by their in-hospital primary clinician and then referred to the research team.

\section{Consent for publication}

Not applicable.

\section{Competing interests}

The authors declare that they have no competing interests.

\section{Publisher's Note}

Springer Nature remains neutral with regard to jurisdictional claims in published maps and institutional affiliations.

\section{Author details}

${ }^{1}$ Duke Global Health Institute, Duke University, Durham, NC, USA. ${ }^{2}$ Duke Emergency Medicine, Duke University School of Medicine, Durham, NC 27710, USA. ${ }^{3}$ Division of Global Neurosurgery and Neuroscience, Duke University, Durham, NC, USA. ${ }^{4}$ UniCesumar, Maringá, Paraná, Brazil. ${ }^{5}$ Duke University School of Medicine, Duke University, Durham, NC, USA.

${ }^{6}$ Kilimanjaro Christian Medical Center, Moshi, Tanzania. ${ }^{7}$ Kilimanjaro Clinical Research Institute, Moshi, Tanzania.

\section{Received: 23 August 2018 Accepted: 26 March 2019} Published online: 08 April 2019

\section{References}

1. Hyder AA, Wunderlich CA, Puvanachandra P, Gururaj G, Kobusingye OC. The impact of traumatic brain injuries: a global perspective. NeuroRehabilitation. 2007;22(5):341-53.
2. Hollis AM, Duncanson H, Kapust LR, Xi PM, O'Connor MG. Validity of the mini-mental state examination and the Montreal cognitive assessment in the prediction of driving test outcome. J Am Geriatr Soc. 2015;63(5):988-92.

3. Langlois JA, Rutland-Brown W, Wald MM. The epidemiology and impact of traumatic brain injury: a brief overview. J Head Trauma Rehabil. 2006; 21(5):375-8.

4. Murray CJ, Lopez AD, Organization WH: The global burden of disease: a comprehensive assessment of mortality and disability from diseases, injuries, and risk factors in 1990 and projected to 2020: summary. 1996.

5. Lovell M, Franzen M. Neuropsychological Assessment. In: Silver JM, Yudofsky SC, Hales RE, editors. Neuropsychiatry of Traumatic Brain Injury. Arlington: American Psychiatric Press; 1994. p. 133-60.

6. Whyte J, Polansky M, Cavallucci C, Fleming M, Lhulier J, Coslett HB. Inattentive behavior after traumatic brain injury. J Int Neuropsychol Soc. 1996;2(04):274-81.

7. Mathias $\mathrm{JL}$, Wheaton P. Changes in attention and information-processing speed following severe traumatic brain injury: a meta-analytic review. In: American Psychological Association; 2007.

8. Hart T, Whyte J, Millis S, Bode R, Malec J, Richardson RN, Hammond F. Dimensions of disordered attention in traumatic brain injury: further validation of the Moss attention rating scale. Arch Phys Med Rehabil. 2006; 87(5):647-55.

9. Levin HS, Goldstein F, High W, Eisenberg H. Disproportionately severe memory deficit in relation to normal intellectual functioning after closed head injury. J Neurol Neurosurg Psychiatry. 1988;51(10):1294-301.

10. Vakil E. The effect of moderate to severe traumatic brain injury (TBI) on different aspects of memory: a selective review. J Clin Exp Neuropsychol. 2005;27(8):977-1021.

11. Stuss D, Ely P, Hugenholtz H, Richard M, LaRochelle S, Poirier C, Bell I. Subtle neuropsychological deficits in patients with good recovery after closed head injury. Neurosurgery. 1985;17(1):41-7.

12. Ruff RM, Levin HS, Mattis S, High WM Jr, Marshall LF, Eisenberg HM, Tabaddor K. Recovery of memory after mild head injury: a three-center study; 1989.

13. MCALLISTER TW. Neurobehavioral sequelae of traumatic brain injury: evaluation and management. World Psychiatry. 2008;7(1):3-10.

14. Deb S, Lyons I, Koutzoukis C. Neuropsychiatric sequelae one year after a minor head injury. J Neurol Neurosurg Psychiatry. 1998;65(6):899-902.

15. Hibbard MR, Uysal S, Kepler K, Bogdany J, Silver J. Axis I psychopathology in individuals with traumatic brain injury. J Head Trauma Rehabil. 1998;13(4):24-39.

16. Koponen S, Taiminen T, Portin R, Himanen L, Isoniemi H, Heinonen $H$, Hinkka S, Tenovuo O. Axis I and II psychiatric disorders after traumatic brain injury: a 30-year follow-up study. Am J Psychiatr. 2002;159(8):1315-21.

17. Max W, MacKenzie EJ, Rice DP. Head injuries: costs and consequences. J Head Trauma Rehabil. 1991;6(2).

18. O'Shanick GJOSA. Personality and Intellectual Changes. In: Silver JM, Yudofsky SC, Hales RE, editors. Arlington: Neuropsychiatry of Traumatic Brain Injury, vol. 163: American Psychiatric Press; 1994. -188.

19. Arciniegas DB, Held K, Wagner P. Cognitive impairment following traumatic brain injury. Curr Treat Options Neurol. 2002;4(1):43-57.

20. Zhang $H$, Zhang XN, Zhang HL, Huang L, Chi QQ, Zhang X, Yun XP. Differences in cognitive profiles between traumatic brain injury and stroke: a comparison of the Montreal cognitive assessment and mini-mental state examination. Chin J Traumatol. 2016;19(5):271-4.

21. Heyman N, Tsirulnicov T, Ben Natan M. Prediction of geriatric rehabilitation outcomes: comparison between three cognitive screening tools. Geriatr Gerontol Int. 2017;17(12):2507-13.

22. Arciniegas DB, Anderson CA, Topkoff J, McAllister TW. Mild traumatic brain injury: a neuropsychiatric approach to diagnosis, evaluation, and treatment. Neuropsychiatr Dis Treat. 2005;1(4):311-27.

23. Folstein MF, Folstein SE, PR MH. "Mini-mental state": A practical method for grading the cognitive state of patients for the clinician. J Psychiatr Res. 1975;12(3):189-98

24. Kosaka B. Neuropsychological assessment in mild traumatic brain injury: a clinical overview. British Columbia Medical Journal. 2006;48(9):447-52.

25. Kaya D, Isik AT, Usarel C, Soysal P, Ellidokuz H, Grossberg GT. The Saint Louis university mental status examination is better than the mini-mental state examination to determine the cognitive impairment in Turkish elderly people. J Am Med Dir Assoc. 2016;17(4).

26. Nasreddine ZS, Phillips NA, Bedirian V, Charbonneau S, Whitehead V, Collin I Cummings JL, Chertkow H. The Montreal cognitive assessment, MoCA: a 
brief screening tool for mild cognitive impairment. J Am Geriatr Soc. 2005; 53(4):695-9.

27. Ozer S, Young J, Champ C, Burke M. A systematic review of the diagnostic test accuracy of brief cognitive tests to detect amnestic mild cognitive impairment. Int J Geriatr Psychiatry. 2016;31(11):1139-50.

28. de Guise E, Leblanc J, Champoux MC, Couturier C, Alturki AY, Lamoureux J, Desjardins M, Marcoux J, Maleki M, Feyz M. The mini-mental state examination and the Montreal cognitive assessment after traumatic brain injury: an early predictive study. Brain Inj. 2013;27(12):1428-34.

29. Wong GK, Ngai K, Lam SW, Wong A, Mok V, Poon WS. Validity of the Montreal cognitive assessment for traumatic brain injury patients with intracranial haemorrhage. Brain Inj. 2013;27(4):394-8.

30. Staton CA, Msilanga D, Kiwango G, Vissoci JR, de Andrade L, Lester R, Hocker M, Gerardo CJ, Mvungi M. A prospective registry evaluating the epidemiology and clinical care of traumatic brain injury patients presenting to a regional referral hospital in Moshi, Tanzania: challenges and the way forward. Int J Inj Control Saf Promot. 2017;24(1):69-77.

31. Katzman R, Zhang M, Ouang Ya Q, Wang Z, Liu WT, Yu E, Wong SC, Salmon DP, Grant I. A Chinese version of the mini-mental state examination; impact of illiteracy in a Shanghai dementia survey. J Clin Epidemiol. 1988;41(10):971-8.

32. Xu G, Meyer JS, Huang Y, Du F, Chowdhury M, Quach M. Adapting mini-mental state examination for dementia screening among illiterate or minimally educated elderly Chinese. Int J Geriatr Psychiatry. 2003; 18(7):609-16.

33. Tanzania National Bureau of Statistics: Tanzania 2012 Census Dashboard. http://www.nbs.go.tz. Accessed 3 Apr 2019.

34. Harris PA, Taylor R, Thielke R, Payne J, Gonzalez N, Conde JG. Research electronic data capture (REDCap) - a metadata-driven methodology and workflow process for providing translational research informatics support. Biomed Inform. 2009;42(2):377-81.

35. Maroco J. Análise de Equações Estruturais: Fundamentos teóricos, software \& Aplicaçōes: Produtos e Servicos de Estatistica, Lda; 2010.

36. Erkut S. Developing multiple language versions of instruments for intercultural research. Child Dev Perspect. 2010;4(1):19-24.

37. Team RC. R: a language and envrionment for statistical computing. $R$ Foundation for Statistical Computing: Vienna; 2014.

38. Kline RB: Principles and Practice of Structural Equation Modeling, Third Edition. New York: Guilford Publications; 2010.

39. Fillenbaum GG, Heyman A, Wilkinson WE, Haynes CS. Comparison of two screening tests in Alzheimer's disease. The correlation and reliability of the mini-mental state examination and the modified blessed test. Arch Neurol. 1987;44(9):924-7.

40. Tinklenberg J, Brooks JO, 3rd, Tanke ED, Khalid K, Poulsen SL, Kraemer HC, Gallagher D, Thornton JE, Yesavage JA: Factor analysis and preliminary validation of the mini-mental state examination from a longitudinal perspective. Int Psychogeriatr 1990, 2(2):123-134.

41. Brugnolo A, Nobili F, Barbieri MP, Dessi B, Ferro A, Girtler N, Palummeri E, Partinico D, Raiteri U, Regesta G, et al. The factorial structure of the mini mental state examination (MMSE) in Alzheimer's disease. Arch Gerontol Geriatr. 2009;49(1):180-5.

42. Castro-Costa E, Fuzikawa C, Ferri C, Uchoa E, Firmo J, Lima-Costa MF, Dewey ME, Stewart R. Dimensions underlying the Mini-Mental State Examination in a sample with low-education levels: the Bambui Health and Aging Study. The Am J Geriatr Psychiatry. 2009;17(10):863-72.

43. Delgado C, Araneda A, Behrens MI. Validación del instrumento Montreal Cognitive Assessment en español en adultos mayores de 60 años. Neurología. 2017.

44. Freitas S, Simões MR, Marôco J, Alves L, Santana I. Construct validity of the Montreal cognitive assessment (MoCA). J Int Neuropsychol Soc. 2012;18(2): 242-50.

45. Duro D, Simoes MR, Ponciano E, Santana I. Validation studies of the Portuguese experimental version of the Montreal cognitive assessment (MoCA): confirmatory factor analysis. J Neurol. 2010;257(5):728-34.

46. Jones RN, Gallo JJ. Dimensions of the mini-mental state examination among community dwelling older adults. Psychol Med. 2000;30(3):605-18.

47. Janelidze M, Mikeladze N, Bochorishvili N, Dzagnidze A, Kapianidze M, Mikava N, Khatiashvili I, Kakhiani D, Mirvelashvili E, Shiukashvili N, et al. Validity of the Georgian Montreal cognitive assessment for the screening of mild cognitive impairment and dementia. Am J Alzheimers Dis Other Dement. 2017;32(1):36-40.
48. Lt H, Bentler PM. Cutoff criteria for fit indexes in covariance structure analysis: conventional criteria versus new alternatives. Struct Equ Model Multidiscip J. 1999;6(1):1-55.

49. Hair JF, Black B, Babin B, Anderson RE, Tatham RL. Multivariate Data Analysis, 6 ed. Upper Saddle River: Pearson Prentice Hall; 2006.

50. DeVellis RF. Scale development: theory and applications. Thousand Oaks: Sage; 2003.

51. Chen KL, Xu Y, Chu AQ, Ding D, Liang XN, Nasreddine ZS, Dong Q, Hong Z, Zhao QH, Guo QH. Validation of the Chinese version of Montreal Cognitive Assessment basic for screening mild cognitive impairment. J Am Geriatr Soc. 2016;64(12):e285-90.

52. Karunaratne $S$, Hanwella R, de Silva V. Validation of the Sinhala version of the Montreal cognitive assessment in screening for dementia. Ceylon Med J. 2011;56(4):147-53

53. Lee JY, Dong Woo L, Cho SJ, Na DL, Hong Jin J, Kim SK, You Ra L, Youn JH, Kwon $\mathrm{M}$, Lee JH, et al. Brief screening for mild cognitive impairment in elderly outpatient clinic: validation of the Korean version of the Montreal cognitive assessment. J Geriatr Psychiatry Neurol. 2008;21(2):104-10

54. Saleh AA, Alkholy R, Khalaf OO, Sabry NA, Amer H, El-Jaafary S, Khali M. Validation of Montreal cognitive assessment-basic in a sample of elderly Egyptians with neurocognitive disorders. Aging Ment Health. 2018;22:1-7.

55. Toglia J, Fitzgerald KA, O'Dell MW, Mastrogiovanni AR, Lin CD. The minimental state examination and Montreal cognitive assessment in persons with mild subacute stroke: relationship to functional outcome. Arch Phys Med Rehabil. 2010;92(5):792-8.

56. Dodds TA, Martin DP, Stolov WC, Deyo RA. A validation of the functional Independence measurement and its performance among rehabilitation inpatients. Arch Phys Med Rehabil. 1993;74(5):531-6.

57. Sweet $L$, Van Adel M, Metcalf $\mathrm{V}$, Wright $\mathrm{L}$, Harley A, Leiva R, Taler $\mathrm{V}$. The Montreal cognitive assessment (MoCA) in geriatric rehabilitation: psychometric properties and association with rehabilitation outcomes. Int Psychogeriatr. 2011;23(10):1582-91.

58. Burton L, Tyson SF. Screening for cognitive impairment after stroke: a systematic review of psychometric properties and clinical utility. J Rehabil Med. 2015:47(3):193-203.

59. Kumar S, Jawahar A, Shah P, Kumar M. Montreal cognitive assessment, a screening tool for mild traumatic brain injury. Neurology. 2015;84(14 Supplement):P7.185.

60. Fujiwara $Y$, Suzuki $H$, Yasunaga $M$, Sugiyama M, ljuin M, Sakuma $N$, Inagaki $\mathrm{H}$, Iwasa $\mathrm{H}$, Ura $\mathrm{C}$, Yatomi $\mathrm{N}$, et al. Brief screening tool for mild cognitive impairment in older Japanese: validation of the Japanese version of the Montreal cognitive assessment. Geriatr Gerontol Int. 2010; 10(3):225-32.

61. Gil L, Ruiz de Sánchez C, Gil F, Romero SJ, Pretelt Burgos F. Validation of the Montreal cognitive assessment (MoCA) in Spanish as a screening tool for mild cognitive impairment and mild dementia in patients over 65 years old in Bogotá, Colombia. Int J Geriatr Psychiatry. 2015;30(6):655-62.

62. Memoria CM, Yassuda MS, Nakano EY, Forlenza OV. Brief screening for mild cognitive impairment: validation of the Brazilian version of the Montreal cognitive assessment. Int J Geriatr Psychiatry. 2013;28(1):34-40.

63. Blunch N. Introduction to structural equation modelling using SPSS and Amos: SAGE Publications; 2008

64. Padilla MA, Divers J. A comparison of composite reliability estimators: coefficient omega confidence intervals in the current literature. Educ Psychol Meas. 2016;76(3):436-53.

65. Rasquin S, van Heugten C, Winkens I, Ritzen W, Hendriksen J, Vles H. Development and validity of the brain injury alert (Bl alert) screening tool for cognitive, emotional and social problems after paediatric acquired brain injury. Brain Inj. 2011;25(7-8):777-86.

66. Hammond FM, Sauve W, Ledon F, Davis C, Formella AE. Safety, tolerability, and effectiveness of dextromethorphan/quinidine for pseudobulbar affect among study participants with traumatic brain injury: results from the PRISM-II open label study. PM R. 2018:10(10):993-1003.

67. Zhang WT, Wang YF. Efficacy of methylphenidate for the treatment of mental sequelae after traumatic brain injury. Medicine (Baltimore). 2017; 96(25):e6960.

68. Su JH, Wu YH, Guo NW, Huang CF, Li CF, Chen CH, Huang MH. The effect of cranioplasty in cognitive and functional improvement: experience of post traumatic brain injury inpatient rehabilitation. Kaohsiung J Med Sci. 2017; 33(7):344-50. 
69. Lee CN, Koh YC, Moon CT, Park DS, Song SW. Serial mini-mental status examination to evaluate cognitive outcome in patients with traumatic brain injury. Korean J Neurotrauma. 2015;11(1):6-10.

70. Ganti L, Daneshvar Y, Ayala S, Bodhit AN, Peters KR: The value of neurocognitive testing for acute outcomes after mild traumatic brain injury. Mil Med Res 2016;3(2095-7467 (Print)):23.

71. Brooks J, Fos LA, Greve KW, Hammond JS. Assessment of executive function in patients with mild traumatic brain injury. J Trauma. 1999; 46(1):159-63.

Ready to submit your research? Choose BMC and benefit from:

- fast, convenient online submission

- thorough peer review by experienced researchers in your field

- rapid publication on acceptance

- support for research data, including large and complex data types

- gold Open Access which fosters wider collaboration and increased citations

- maximum visibility for your research: over $100 \mathrm{M}$ website views per year

At $\mathrm{BMC}$, research is always in progress.

Learn more biomedcentral.com/submissions 